\title{
PAUL MARTIN
}

\section{ANDRÉ MERLIN}

\section{Méthode combinatoire de répartition à court terme d'un ensemble de moyens de production thermique hydraulique}

Revue française d'automatique, d'informatique et de recherche opérationnelle. Recherche opérationnelle, tome 4, n V2 (1970), p. 77-96.

<http://www.numdam.org/item?id=RO_1970_4_2_77_0>

(C) AFCET, 1970, tous droits réservés.

L'accès aux archives de la revue « Revue française d'automatique, d'informatique et de recherche opérationnelle. Recherche opérationnelle » implique l'accord avec les conditions générales d'utilisation (http://www.numdam.org/ legal.php). Toute utilisation commerciale ou impression systématique est constitutive d'une infraction pénale. Toute copie ou impression de ce fichier doit contenir la présente mention de copyright.

\section{Numdam}


R.I.R.O.

(4 $4^{\mathrm{e}}$ année, V-2, 1970, p. 77-96)

\title{
METHODE GOMBINATOIRE DE RÉPARTITION A GOURT TERME D'UN ENSEMBLE DE MOYENS DE PRODUGTION THERMIQUE HYDRAULIQUE
}

\author{
par MM. Paul Martin et André Merlin ( ${ }^{1}$ )
}

Résumé. - On expose ici un modèle combinatoire pour résoudre le problème à court terme visant à trouver le plan de production optimal pour un parc de production hydraulique et thermique.

Cet algorithme a été utilisé pour un réseau de transport à 200 nœuds et se sert d'une méthode de programmation mathématique du type "branch and bound" jointe à une méthode de gradient.

1. Exposé DU PROBLEME

\section{PLAN}

2. Formulation mathematique

3. ChotX DES GROUPES SUR UN PALIER DE PRODUCTION SANS RESEAU

4. Choix des groupes Sur UN Palier de production aVec Reseau

5. Choix Des groupes thermiques et hydrauliques SUR UN ensemble de paliers de production avec reseau

6. Utilisation DES RESUltats

\section{INTRODUCTION}

La variation journalière d'électricité nécessite la détermination d'un planning de marche de chaque centrale dont la puissance est modulable au cours de la journée. Le but de ce planning est de satisfaire la demande d'énergie au moindre coût de combustible selon les règles de sécurité que l'on s'est fixées.

On dispose de centrales hydrauliques et thermiques aux caractéristiques techniques et économiques différentes.

(1) Électricité de France, Direction des Études et Recherches, Service Etudes de Réseaux. 
Un groupe hydraulique est disponible à tout instant. Il peut fonctionner à une puissance comprise entre 0 et sa puissance maximale. L'eau est gratuite mais en quantité limitée.

L'utilisation d'un groupe thermique entraîne des charges fixes : il faut payer sans produire de l'énergie, uniquement pour l'amener au démarrage ; il ne peut fonctionner au-dessous d'un minimum technique. Les frais de combustible (charbon ou fuel) sont proportionnels à la puissance de marche.

On est donc amené à considérer des variables de deux sortes :

- des variables bivalentes $y(0,1)$ indiquant l'arrêt ou la marche d'un groupe thermique,

- des variables continues $P$ et $H$ indiquant le niveau de puissance des groupes thermiques et hydrauliques.

Il faut résoudre un programme mathématique en variables mixtes qui rend compte de la structure combinatoire du problème.

$\mathrm{Au}$ voisinage d'une solution réalisable, on sépare l'optimisation du thermique de celle de l'hydraulique.

Le planning thermique est déterminé par une méthode de séparation et d'évaluation progressive (méthode Branch and Bound). Puis l'hydraulique est ajusté par la minimisation d'une fonction non linéaire sous des contraintes linéaires. On procède par linéarisations successives de la fonction économique.

L'optimisation hydraulique peut amener à réviser le planning thermique initial. Des itérations sont alors nécessaires entre les deux phases de calcul.

\section{EXPOSE DU PROBLEME}

On veut déterminer, pour un ensemble de groupes thermiques et hydrauliques disponibles à un instant donné, le diagramme de fonctionnement de chacun d'eux pendant une période de temps qui est en général la journée, afin que le coût total de production (coût de démarrage des unités thermiques inclus) soit minimum, la consommation étant satisfaite et l'énergie hydraulique à turbiner pour cette période étant connue par avance, en chaque sommet du réseau de transport.

Il faut donc optimiser à cette fin, les instants d'arrêt et de démarrage de chaque groupe, ainsi que le niveau de puissance produite par chacun d'eux à chaque instant.

On rappelle les hypothèses que l'on doit faire :

\section{Hypothèse 1 :}

Caractéristiques technico-économiques des groupes thermiques.

Le coût horaire de production $\gamma_{i}$ est une fonction linéaire de la puissance produite $P_{i}$

$$
\gamma_{i}=a_{i}+b_{i} \cdot P_{i}
$$


$a_{i}$ représente le coût de marche à vide du groupe $i$,

$b_{i}$ représente son coût marginal de production.

Une telle hypothèse n'est pas restrictive, du moins pour la plupart des groupes français.

La puissance d'un groupe thermique peut être nulle ou comprise entre deux valeurs son minimum technique $\underline{P}_{i}$ et sa puissance maximum $\bar{P}_{i}$. Afin de distinguer ces deux états, on introduit une variable $y_{i, t}$ qui peut prendre deux valeurs :

$y_{i, t}=0 \quad$ Le groupe $i$ est arrêté à l'instant $t P_{i, t}=0$

$y_{i, t}=1$ Le groupe est en marche $\underline{P}_{i} \leqslant P_{i, t} \leqslant \bar{P}_{i}$

Soit sous une forme plus algébrique :

$$
y_{i, t} P_{i} \leqslant P_{i, t} \leqslant y_{i t} \bar{P}_{i} \quad y_{, t}=(0,1)
$$

Lorsque l'on arrête un groupe thermique, les pertes calorifiques dues à un refroidissement de la chaudière pénalisent un redémarrage éventuel, car il est nécessaire de dépenser une certaine énergie pour placer l'unité en l'état de fonctionner. Cette dépense est fonction croissante du temps d'arrêt $\tau$. Plusieurs types de fonction ont été proposés. On adopte en général une fonction exponentielle à laquelle on adjoint un terme constant pour rendre compte des différentes charges fixes dues à un arrêt.

$$
C d(\tau)=C d^{0}\left(1-\mathrm{e}^{-w \cdot \tau}\right)+f^{0}
$$

D'autre part afin d'éviter plusieurs arrêts consécutifs d'un même groupe au cours d'une journée, ce qui provoquerait une fatigue trop importante du matériel, on le contraint à s'arrêter au plus une fois en $24 \mathrm{~h}$. Ce qui s'exprime simplement par l'inégalité :

$$
\sum_{i=1}^{23}\left(y_{i, t}-y_{i, t+1}\right)^{2} \leqslant 2
$$

$y_{i, t}$ représente comme précédemment l'état du groupe $i$ sur le palier $t$.

\section{Hypothèse 2 :}

Caractéristiques des groupes hydrauliques.

La puissance d'un groupe hydraulique $H_{i, t}$ est comprise entre une valeur maximale et une valeur nulle. Cette hypothèse se justifie dans la mesure où le minimum technique d'un tel groupe est voisin de zéro.

$$
0 \leqslant H_{i, t} \leqslant \vec{H}_{i}
$$

Une quantité d'énergie hydraulique est à utiliser au cours de la journée, en chaque nœud du réseau où il existe des lacs et des éclusées dont la production n'est pas encore imposée à chaque instant. Ces quantités $E_{i}$ sont fixées par avance grâce à un programme de gestion des réservoirs. 
On doit done avoir :

$$
\sum_{t} H_{i, t}=E_{i} \forall i
$$

\section{Hypothèse 3 :}

Le réseau électrique est introduit sous la forme des relations d'injection écrites en "actif " seul. La tension est donc fixée en chaque nœud en module. Seul le déphasage de ces tensions par rapport à celle d'un nœud de référence est susceptible de varier.

On notera ces relations :

$$
P_{i, t}+H_{i, t}-C_{i, t}=\varphi_{i}\left(\theta^{t}\right) \quad \forall i, t
$$

$C_{i, t}$ représente la consommation au nœud $i$ à l'instant $t$

$\theta_{i}^{t}$ représente le déphasage de la tension au nœud $i$ à l'instant $t$.

Ces relations seront explicitées par la suite.

On supposera en outre que les contraintes de transit sont toutes satisfaites.

\section{Hypothèse 4 :}

Contraintes de réserve tournante.

Afin de respecter la règle de sécurité $n-1$ on assure sur chaque palier horaire une réserve de puissance thermique égale à la puissance maximale du groupe le plus puissant, ce qui permet de remédier rapidement à la défaillance de l'un quelconque des groupes en service.

Si $R_{t}^{0}$ est cette valeur, on doit avoir :

$$
\sum_{i} \bar{P}_{i} \cdot y_{i, t}-\sum_{i} P_{i, t} \geqslant R_{t}^{0} \quad \forall t
$$

Cette réserve tournante est une réserve globale de nature thermique qui ne tient pas compte des contraintes éventuelles introduites par le réseau. On peut la régionaliser en sommant sur les indices correspondant à un sous-ensemble de sommets du réseau. On peut également introduire une contrainte de réserve tournante mixte hydraulique et thermique de la forme suivante :

$$
\sum_{i}\left(\bar{H}_{i}-H_{i, t}\right)+\sum_{i} \bar{P}_{i} \cdot y_{i, t}-\Sigma P_{i, t} \geqslant R_{t}^{0} \quad \forall t
$$

\section{Hypothèse 5 :}

La journée que l'on considère est une journée type (mercredi). Les courbes de charge aux différents nœuds du réseau sont supposées périodiques et de même période 24 heures.

Dans le cas contraire, il est nécessaire de préciser l'état de marche ou d'arrêt des groupes à l'instant initial et à l'instant final. 


\section{FORMULATION MATHEMATIQUE DU PROBLEME}

On reprendra les notations introduites précédemment. Afin de simplifier les notations et à cette seule fin, on supposera qu'il y a au plus un groupe thermique et un groupe hydraulique par sommet du réseau.

L'indice $i$ représente le numéro du sommet.

L'indice $t$ représente le numéro du palier horaire.

Minimiser $F=\sum_{i, t} a_{i} y_{i, t}+b_{i} p_{i, t}+\sum_{i} C d_{i}\left(\tau_{i}\right)$

$$
\left\{\begin{array}{l}
P_{i, t}+H_{i, t}-C_{i, t}=\varphi_{i}\left(\theta^{t}\right) \quad \forall i, t \\
\sum_{t} H_{i, t}=E_{i} \\
\sum_{t=1}^{23}\left(y_{i, t+1}-y_{i, t}\right)^{2} \leqslant 2 \quad \forall i \\
\sum_{i} \bar{P}_{i} y_{i, t}-\sum_{i} P_{i, t} \geqslant R_{t}^{0} \quad \forall t \quad \forall i \\
\operatorname{avec} y_{i, t}=(0,1) \quad \tau_{i}=\sum_{t}\left(1-y_{i, t}\right) \quad \forall i \quad \text { si } \tau_{i}=24 \\
y_{i, t} P_{i} \leqslant P_{i, t} \leqslant y_{i, t} \bar{P}_{i} \\
\quad 0 \leqslant H_{i, t} \leqslant \bar{H}_{i} \quad \text { si } \tau_{i}<24
\end{array}\right.
$$

Ainsi posé, ce programme mathématique est non linéaire à la fois par rapport aux contraintes et par rapport à la fonction à optimiser. Il se formule en variables mixtes (variables continues et variables bivalentes) ce qui montre le caractère combinatoire du problème traité. L'algorithme proposé utilisera une méthode de séparation et d'évaluation progressive (méthode Branch and Bound) particulièrement adaptée à ces types de programmes.

Pour simplifier l'exposé d'ensemble, on traitera tout d'abord le problème du choix des groupes thermiques sur un palier de production dans lequel on néglige l'influence des coûts de démarrage et du réseau de transport ainsi que les contraintes de réserve tournante.

Puis on traitera ce même problème avec réseau de transport en utilisant les hypothèses précisées ci-dessus.

On abordera enfin le problème complet sur une journée, avec un ensemble thermique hydraulique, coûts de démarrage et réserve tournante inclus. 


\section{CHOIX DES GROUPES SUR UN PALIER DE PRODUCTION, SANS RESEAU}

\subsection{Enoncé du problème}

On cherche parmi l'ensemble des $n$ groupes disponibles, le sousensemble qui satisfera la consommation thermique $\Gamma$ demandée au cout minimum.

En utilisant les notations posées ci-dessus, on obtient le programme suivant :

(2)

$$
\begin{aligned}
& \text { Minimiser } \sum_{i=1}^{n} a_{i} y_{i}+b_{i} P \\
& \left\{\begin{array}{l}
y_{i} \underline{P}_{i} \leqslant P_{i} \leqslant y_{i} \bar{P}_{i} \\
\Sigma P_{i}=\Gamma \\
y_{i}=\left\{\begin{array}{l}
0 \\
1
\end{array}\right.
\end{array}\right.
\end{aligned}
$$

C'est un programme linéaire en variables mixtes. Le domaine des contraintes n'est pas convexe comme on le voit sur la représentation graphique d'un exemple à deux variables.

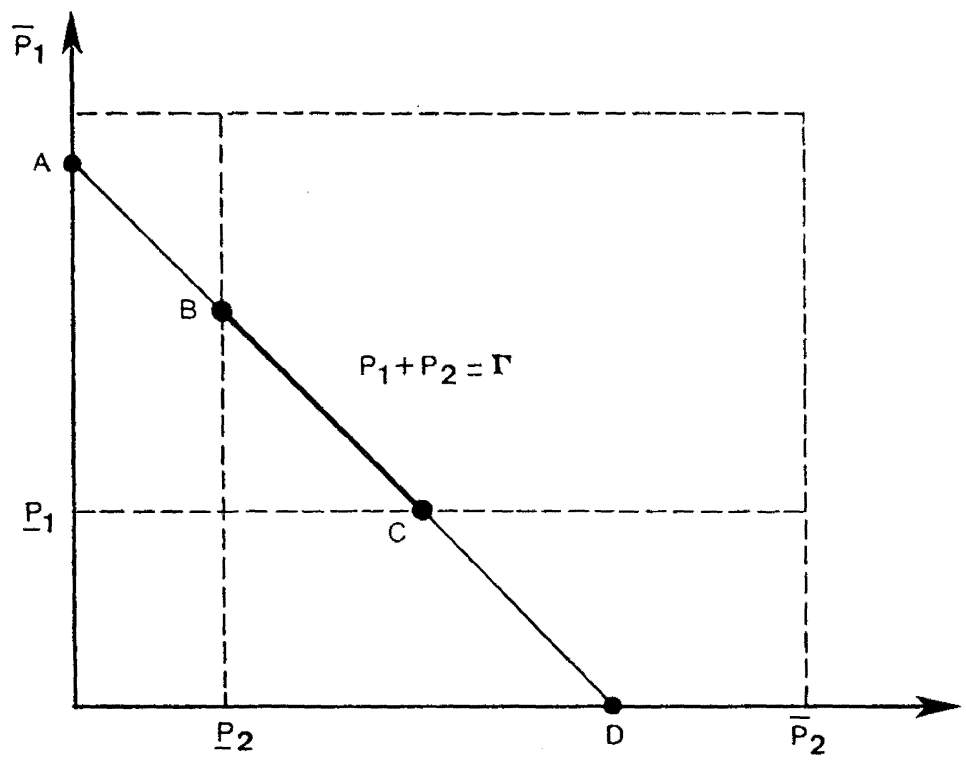

Figure 1 
Ce domaine est ici constitué par les points $A$ et $D$ et le segment de droite $B C$.

\subsection{L'outil mathématique :}

La procédure S.E.P. (séparation et évaluation progressive)

Sans vouloir reprendre la théorie de cette méthode, on va montrer comment il est possible de l'appliquer à cette classe de problèmes linéaires où les variables entières sont bivalentes.

Soit $I_{0}$ l'ensemble ordonné des nombres entiers de 1 à $n$.

Soit

$$
A_{p}=\left(y_{i 1}, y_{i 2}, \ldots, y_{i_{r}}\right) \quad 0 \leqslant r \leqslant n
$$

un ensemble de $r$ valeurs égales à 0 ou 1 .

$\left(i_{1}, i_{2}, \ldots, i_{r}\right)$ représentent un sous-ensemble $I_{r}$ lui-même ordonné de l'ensemble ordonné $I_{0}$. $A_{p}$ est donc un sommet du cube unité dans l'espace à $r$ dimensions.

Supposons que l'on ait fixé la valeur de ces $r$ variables, et essayons de résoudre le problème ainsi posé dans le domaine défini par le cube des variables bivalentes non encore fixées.

Le problème exprimé en (2) s'écrit :

$$
\text { Minimiser } \sum_{k=1}^{r} a_{i k} y_{i k}^{*}+b_{i k} P_{i k}+\sum_{l=r+1}^{n} a_{i l} y_{i l}+b_{i l} P_{i l}
$$

$$
\left\{\begin{array}{l}
\sum_{k=1}^{n} P_{i k}=\Gamma \\
y_{i t}=\left\{\begin{array}{l}
0 \\
1
\end{array} \quad y_{i k}^{*}=\left\{\begin{array}{l}
0 \\
1
\end{array}\right.\right. \\
y_{i k}^{*} \underline{P}_{i k} \leqslant P_{i k} \leqslant y_{i k}^{*} \bar{P}_{i k} \\
y_{i k} \underline{P}_{i l} \leqslant P_{i l} \leqslant y_{i l} \bar{P}_{i l}
\end{array}\right.
$$

Le signe ${ }^{*}$ permet de distinguer les variables bivalentes déjà fixées de celles qui ne le sont pas encore.

Le minimum $\varphi_{p}$ de (3) est supérieur ou égal au minimum $\varphi_{0}$ de (2) puisque l'espace des solutions réalisables du premier programme est contenu dans celui du second. En effet (3) a été obtenu à partir de (2) en fixant la valeur de certaines variables $y_{i k}$ à 0 ou à 1 .

Le problème (3) ne semble pas plus facile à résoudre que (2); toutefois on va montrer qu'il est aisé d'encadrer, et c'est là un trait important de la procédure S.E.P., l'optimum $\varphi_{p}$ entre deux valeurs : une valeur par 
défaut $V d_{p}$ qui est une solution optimale du programme $\left(3^{\prime}\right)$ et $V r_{p}$, valeur par excès qui est une solution réalisable du programme (3).

\subsubsection{L'évaluation par défaut}

Le programme $\left(3^{\prime}\right)$ que l'on résout pour obtenir $V d_{p}$ est le suivant :

$$
\begin{aligned}
& \text { Minimiser } \sum_{k=1} a_{i k} y_{i k}^{*}+b_{i k} P_{i k}+\sum_{l=r+1}^{n} \gamma_{i l} P_{i l}
\end{aligned}
$$

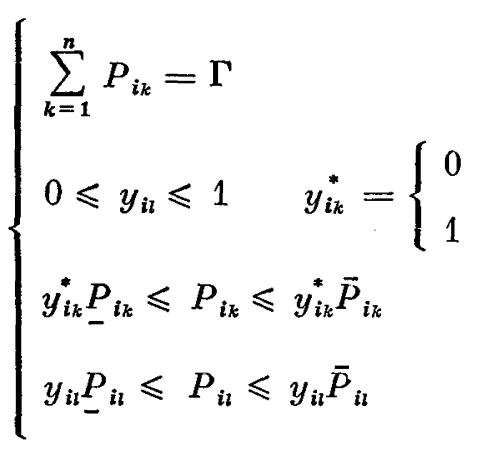

La dernière contrainte peut encore s'écrire :

$$
0 \leqslant P_{i l} \leqslant \bar{P}_{i l}
$$

puisque

$$
0 \leqslant y_{i l} \leqslant 1
$$

Le domaine défini par les contraintes de $\left(3^{\prime}\right)$ est convexe, et il contient le domaine des contraintes du programme (3).

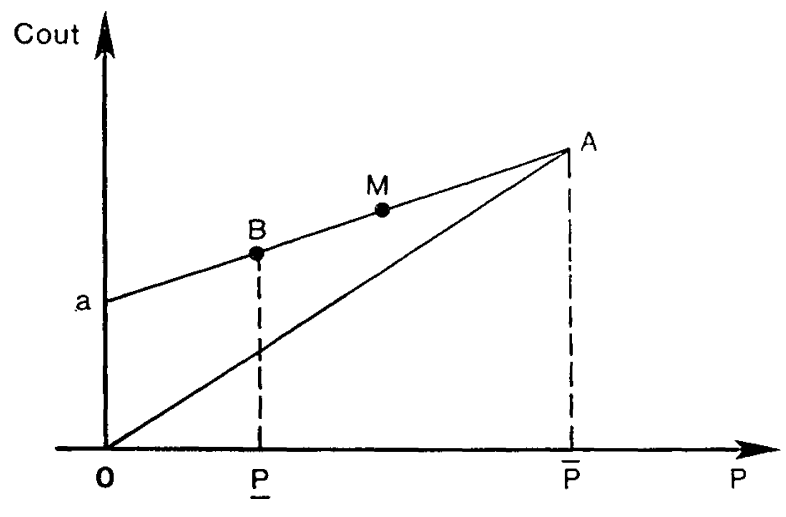

Figure 2

Si $\gamma_{i l}$ est le coût moyen du groupe $i_{l}$ calculé à puissance maximum, on voit sur le figure 2 que $\gamma_{i b}$ est inférieur ou égal au coût moyen réel du 
groupe, quel que soit son niveau de puissance (la pente de $O M$ est supérieure ou égale à la pente de $O A$ quel que soit $M$ sur le segment $A B$ ).

La fonction économique de (3) est donc minorée par celle de $\left(3^{\prime}\right)$.

Il est facile de trouver l'optimum de $\left(3^{\prime}\right)$ puisqu'il s'agit d'un programme linéaire à variables continues bornées avec une seule contrainte d'égalité du type suivant :

$$
\text { Minimiser } \begin{cases}\Sigma \alpha_{i} x_{i} \quad & \alpha_{i} \geqslant 0 \\ \Sigma x_{i}=y \quad y \geqslant 0 & y \leqslant x_{i} \leqslant a_{i}\end{cases}
$$

Il suffit pour ce faire, de prendre les variables $x_{i}$ dans l'ordre des $\alpha_{i}$ croissants à leur maximum $a_{i}$ jusqu'à ce que la demande $y$ soit satisfaite. La dernière choisie sera la variable qui assure l'ajustement de l'offre à la demande.

\subsubsection{L'évaluation par excès}

La valeur $V d_{p}$ de l'évaluation par défaut ayant été déterminée, il s'agit de trouver une solution réalisable $V r_{p}$ dans $A_{p}$ aussi proche que possible de $V d_{p}$, afin que l'optimum du problème (3), soit encadré au mieux par ces deux valeurs. Pour obtenir cette solution, on choisit les mêmes groupes que ceux utilisés pour obtenir $V d_{p}$ mais la répartition des puissances entre ces divers groupes est faite selon les coûts marginaux croissants. Chaque groupe est au moins à son minimum technique et sa puissance est élevée à sa valeur maximum dans l'ordre des coûts marginaux $b_{i}$ croissants, jusqu'à ce que la consommation totale soit satisfaite.

Ainsi a-t-on associé à un sous-ensemble $A_{p}$ du cube des variables bivalentes, deux scalaires $V r_{p}$ et $V d_{p}$ tels que l'optimum $\varphi_{p}$ du problème (3) sur $A_{p}$ soit compris entre ces deux valeurs

$$
V d_{p} \leqslant \varphi_{p} \leqslant V r_{p}
$$

\subsubsection{L'algorithme}

Soit $A_{0}$ l'ensemble initial où aucune des variables bivalentes n'a été fixée. A cet ensemble seront associés les deux scalaires $V d_{0}$ et $V r_{0}$. Selon un critère de séparation qui sera précisé par la suite, une variable bivalente $y_{i 0}$ est choisie pour être l'objet d'un arbitrage.

L'ensemble $A_{0}$ est séparé en deux sous-ensembles $A_{1}$ et $A_{2}$ tels que dans l'un $y_{i 0}=0$ et dans l'autre $y_{i 0}=1$, et tels que :

$$
A_{1} \cup A_{2}=A_{0}
$$

A l'ensemble $A_{1}$, on associe $V d_{1}$ et $V r_{1}$.

A l'ensemble $A_{2}$, on associe $V d_{2}$ et $V r_{2}$. 
On a nécessairement :

$$
\begin{aligned}
& V d_{1} \geqslant V d_{0} \\
& V d_{2} \geqslant V d_{0}
\end{aligned}
$$

On choisit parmi ces deux sous-ensembles celui qui a l'évaluation par défaut minimum. On suppose en effet que l'exploration sera la plus efficace dans le sous-ensemble ayant l'évaluation par défaut la plus petite. (On n'élimine pas a priori le deuxième sous-ensemble.)

Le sous-ensemble étant choisi, on renouvelle le processus. Ainsi à l'étape $p$, si l'on figure chaque sous-ensemble $A_{r}$ par un point, l'ensemble de la procédure peut être représentée par une arborescence dont les sommets pendants sont les sous-ensembles non encore arbitrés et dont les branches figurent les séparations successives.

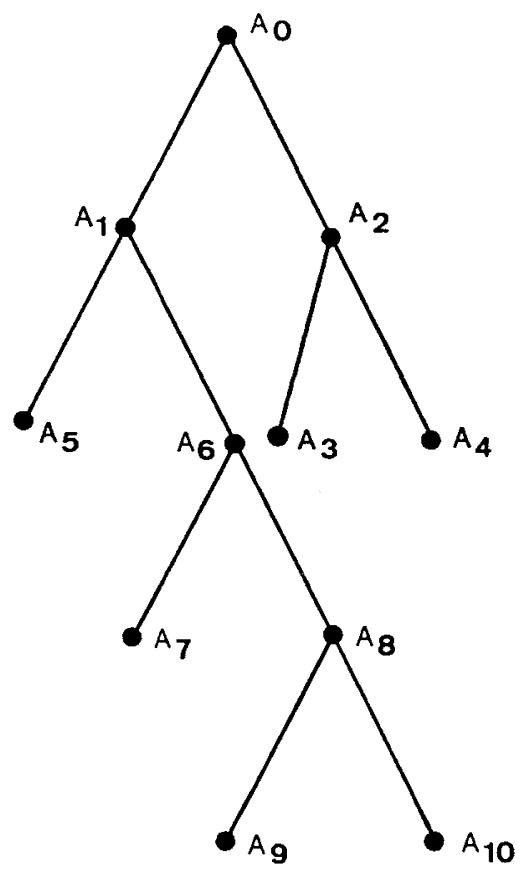

Figure 3

\subsubsection{Critères d'élimination et d'arrêts}

Si l'on désigne par $V r^{*}$, la plus petite valeur des solutions réalisables déjà calculées et si pour un sommet pendant $A_{q}$, on obtient une évaluation par défaut $V d_{q}$ telle que :

$$
V d_{q}>V r^{*}
$$

.Il est inutile d'explorer les sommets issus de ce sommet. En effet lorsque l'on chemine de la racine de l'arborescence vers une extrémité 
l'évaluation $V d_{q}$ est non décroissante. Il en résulte que pour tout sommet issu de $A_{q}$, l'évaluation par défaut relative à ce sommet est supérieure à la solution réalisable $V r^{*}$, donc a fortiori toute solution réalisable associée à ce sommet. Le sommet $A_{q}$ est donc à éliminer.

Si pour un sous-ensemble $A_{q}$, les deux scalaires associés sont tels que : $V d_{q}=V r_{q}$ le sommet représentant $A_{q}$ est terminal. Il est inutile de poursuivre l'exploration plus avant à partir de ce sommet puisque l'optimum $\varphi_{a}$ sur ce sous-ensemble compris entre ces deux valeurs, est égal dans ce cas à leur valeur commune.

Si $A_{q}$ est vide, il est également terminal.

Ainsi à une étape de la procédure, l'arborescence possède un ensemblè de sommets candidats non éliminés et non terminaux pouvant donner lieu chacun à une nouvelle exploration.

\subsubsection{Le principe de séparation}

Le sommet choisi parmi l'ensemble des sommets candidats sera celui qui a l'évaluation par défaut minimale comme il a été dit ci-dessus.

Puis il faut fixer la variable bivalente à arbitrer parmi l'ensemble des variables bivalentes non encore arbitrées au sommet choisi. Ce choix peut être déterminé par un ordre $a$ priori. Mais il peut aussi résulter des valeurs prises par une fonction pénalité attribuée à chacune de ces variables en ce sommet. La variable choisie correspondra à la pénalité minimum.

La variable bivalente ayant été déterminée, on impose sa valeur à 0 puis à 1 ; ce qui donne deux nouveaux sommets auxquels on associe les deux scalaires : évaluation par défaut et évaluation par excès.

La procédure ne s'arrêtera que lorsque tous les sommets pendants seront terminaux. Cet événement aura lieu après un nombre fini d'étapes puisque le nombre de variables bivalentes est lui-même fini. La séparation sera d'autant meilleure que le nombre de sommets de l'arborescence explorés sera plus faible.

\section{CHOIX DES GROUPES SUR UN PALIER DE PRODUCTION AVEC RESEAU}

Seules seront prises en compte les productions actives des groupes. Les contraintes de transit seront négligées.

Les tensions sont donc fixées en module. Et les déphasages de ces tensions par rapport au nœud de référence ne sont pas bornés.

Les notations que l'on utilisera dans ce paragraphe sont celles qui sont utilisées pour le "dispatching économique » ou les méthodes de calcul de répartition des transits sur un réseau de transport. 


\subsection{Les équations du réseau}

$$
\begin{array}{ll}
I_{i} \equiv P_{i}-C_{i}=\varphi_{i}(\theta) & \forall i=1, \ldots, n \text { indice } \\
& \text { du nœud du réseau }
\end{array}
$$

avec :

$$
\varphi_{i}(\theta)=V_{i}^{2} \sum_{j \in \alpha(i)}\left(\frac{\cos \xi_{i j}}{Z_{i j}}+g_{i j}\right)-V_{i} \sum_{j \in \alpha(i)} \frac{V_{j}}{Z_{i j}} \cos \left(\xi_{i j}+\theta_{i}-\theta_{j}\right)
$$

$\alpha(i)$ : ensemble des sommets voisins de $i$.

Le jacobien du système (1) est une matrice carrée d'ordre $n$ et de rang $(n-1)$. On montre en effet que l'on a l'égalité

$$
\sum_{j=1}^{n} \varphi_{i j}^{\prime}=0 \quad \forall i=1, \ldots, n
$$

Il existe par conséquent une relation linéaire entre les vecteurs $\left(\varphi_{i}^{\prime}\right)$ que l'on peut écrire :

$$
\sum_{i=1}^{n} \lambda_{i} \varphi^{\prime}(\theta)=0
$$

Or :

$$
\mathrm{d} P_{i}=<\varphi_{i}^{\prime}(\theta), \mathrm{d} \theta>
$$

égalité obtenue en différenciant (1).

D'où la relation fondamentale

$$
<\lambda, \mathrm{d} P>=0
$$

\subsection{Enoncé du problème}

On pose :

$$
P_{i}=\sum_{k=1}^{g(i)} P_{i}^{k}
$$

$g(i)$ représente le nombre de groupes rattachés au nœud $i$.

Soit à résoudre le programme mathématique :

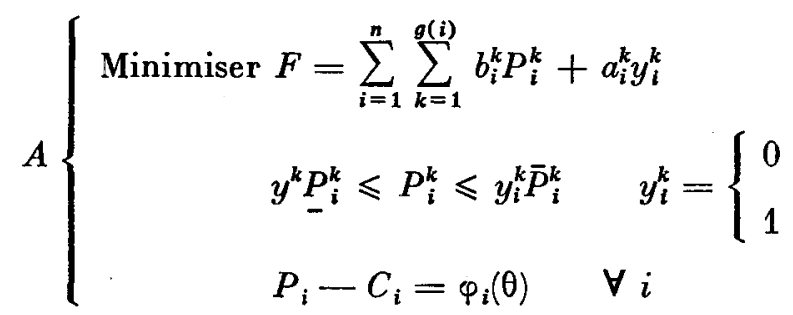

La dernière contrainte relative aux pertes est non linéaire. Afin de pouvoir appliquer la méthode exposée au paragraphe 3 , on linéarisera 
cette équation au voisinage d'une solution réalisable. Or, il est facile de montrer qu'on aboutit à la relation (2) :

$$
<\lambda, \mathrm{d} P>=0
$$

qui s'écrit alors :

$$
\sum_{i=1}^{n} \lambda_{i}^{(0)} P_{i}=K=\Sigma \lambda_{i}^{(0)} P_{i}^{0}
$$

On résout ainsi le programme suivant :

$$
B\left\{\begin{array}{c}
\text { Minimiser } F=\Sigma \Sigma b_{k}^{k} P_{i}^{k}+a_{i}^{k} y_{i}^{k} \\
y_{i}^{k} P_{i}^{k} \leqslant P_{i}^{k} \leqslant y_{i}^{k} \bar{P}_{i}^{k} \\
\Sigma \lambda_{i}^{(0)} P_{i}=K
\end{array} \quad y_{i}^{k}=\left\{\begin{array}{c}
0 \\
1
\end{array}\right.\right.
$$

Sa structure est identique à la structure de celui qui a été résolu au paragraphe 3. Par conséquent le même algorithme s'applique.

\subsection{Influence des termes d'ordre supérieur à 1 , dans les équations du réseau, sur le planning thermique}

Soit $\left(y_{i(p)}^{\left.k(n)^{*}\right)}\right.$ la solution optimale du programme $B$ obtenue à partir de $\left(\lambda_{i(p-1)}^{(n)}\right)$.

On associe à ce planning le coût d'exploitation $\psi_{(p)}^{(n)}$ correspondant à la répartition des puissances thermiques à coût minimal (l'état de marche ou d'arrêt de tous les groupes est alors fixé) ; ce coût est obtenu en résolvant le programme suivant :

$$
C\left\{\begin{array}{l}
\operatorname{Min} \sum_{i=1}^{n} \sum_{k=1}^{g(i)} a_{i}^{k} y_{i(p)}^{k(n)^{*}}+b_{i}^{k} P_{i}^{k} \\
y_{i(p)}^{k(n) *} \underline{P}_{i}^{k}<P_{i}^{k}<y_{i(p)}^{k(n)} \bar{P}_{i}^{k} \\
\sum_{k=1}^{g(i)} P_{i}^{k}-C_{i}=\varphi_{i}(\tilde{\theta})
\end{array}\right.
$$

Le programme $C$ est un dispatching économique en "actif » seul dont la résolution est connue.

Soit $\psi_{0}^{(n)}$ la meilleure solution trouvée à cette étape de l'algorithme. A cette solution sont associées les valeurs duales $\lambda_{i(0)}^{(n)}$.

1. Les paramètres $\left(\lambda_{i}\right)$ étant égaux à $\left(\lambda_{i(p)}^{(n)}\right)$ la résolution du programme $B$ permet de déterminer un nouveau planning thermique défini par les variables d'état $\left(y_{i(p+1)}^{k(n)}\right)$. 
2. On résout le programme $C$; ce qui donne les valeurs $\psi_{(p+1)}^{(n)}$ et $\left(\lambda_{i(p+1)}^{(n)}\right)$

3. a) $\mathrm{Si}$

$$
\begin{aligned}
& \psi_{(p+1)}^{(n)}<\psi_{(0)}^{(n)} \\
& \text { on pose : } \psi_{(0)}^{(n+1)}=\psi_{(p+1)}^{(n)} \\
& \lambda_{(0)}^{(n+1)}=\lambda_{(p+1)}^{(n)}
\end{aligned}
$$

$p=0$

$n=n+1$ et l'on revient en 1 .
b) $\mathrm{Si}$
$\psi_{(p+1)}^{(n)} \geqslant \psi_{(0)}^{(n)}$

On pose :

$$
y_{i(p+2)}^{k(n)^{*}}=y_{i(0)}^{k(n)^{*}} \quad \text { si } \quad y_{i(p+1)}^{k(n)^{*}}=y_{i(0)}^{k(n) *}
$$

dans le cas contraire, on n'impose pas la valeur de $y_{i(p+2)}^{k(n)}$.

On résout $B$ en éliminant a priori la solution $\left(y_{i(p+1)}^{k(n) *}\right)$

On pose $p=p+1$ et l'on revient en 2 .

Lorsque toutes les variables $y_{i}^{k}$ sont fixées et égales à $y_{i(0)}^{k(n)}$ on a atteint un optimum local du problème $A$.

\section{CHOIX DES GROUPES THERMIQUES ET HYDRAULIQUES SUR UN ENSEMBLE DE PALIERS DE PRODUCTION AVEC RESEAU}

On traite ici le problème dans toute sa généralité tel qu'il est formulé dans le deuxième paragraphe.

Comme il a été fait précédemment, on linéarise les équations du réseau au voisinage d'une solution réalisable.

On obtient le problème suivant :

$$
\begin{gathered}
\text { Minimiser } \sum_{t} \sum_{i} a_{i} y_{i, t}+b_{i} P_{i, t}+\sum_{i} C d_{i}\left(\tau_{i}\right) \\
\begin{cases}y_{i, t} P_{i} \leqslant P_{i, t} \leqslant y_{i, t} \bar{P}_{i} & \tau_{i}=\sum_{t}\left(1-y_{i, t}\right) \\
0 \leqslant H_{i, t} \leqslant \bar{H}_{i} & \left(y_{i, t}-y_{i, t+1}\right)^{2} \leqslant 2 \\
\sum_{i} y_{i, t} \bar{P}_{i}-\sum_{i} P_{i, t} \geqslant R_{t}^{0} & y_{i, t}=\left\{\begin{array}{l}
0 \\
1 \\
\sum_{i} H_{i, t}=E_{i} \quad \forall i
\end{array}\right. \\
\sum_{i} \lambda_{i, t}^{0}\left(P_{i, t}+H_{i .}\right)=K_{t}^{0} & \forall t\end{cases}
\end{gathered}
$$


Ainsi posé, le problème peut être résolu simplement car il est facile de montrer que les variables hydrauliques prennent des valeurs qui sont fixées indépendamment des valeurs prises par $P_{i, t}$ (compte tenu de cette linéarisation, et si l'on se fixe la valeur des variables $\left.y_{i, t}\right)$.

En un même nœud l'utilisation de l'hydraulique sera d'autant plus forte que le coût de l'énergie $\lambda_{i, t}^{0}$ sera plus grand.

L'hydraulique au nœud $i$ sera utilisée à puissance maximale dans l'ordre du $\lambda_{i, t}^{0}$ décroissant jusqu'à satisfaire l'énergie $E_{i}$ imposée.

Il est donc possible après avoir linéarisé les équations du réseau de séparer l'optimisation du thermique de celle de l'hydraulique puisque les coûts $\lambda_{i, t}^{0}$ sont alors fixés.

\subsection{Optimisation du planning des groupes thermiques}

Il s'agit de :

$$
\begin{aligned}
& \text { Minimiser } \sum_{t} \sum_{i} a_{i} y_{i, t}+b_{i} P_{i, t}+\sum_{i} C d_{i}\left(\tau_{i}\right)
\end{aligned}
$$

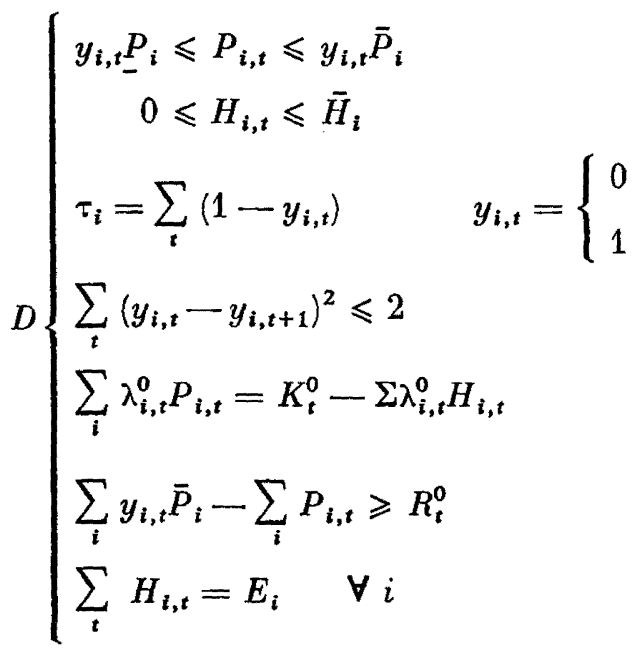

La méthode utilisée est celle proposée au paragraphe 3.

Supposons qu'en un sommet de l'arborescence $r$ variables bivalentes soient fixées, l'optimum d'un tel programme sera encadré par deux valeurs que l'on calculera. L'évaluation par défaut $V d$ est obtenue en résolvant le programme linéaire continu suivant, dans lequel les variables $H_{t, t}^{0}$ sont fixées comme il a été dit précédemment. 


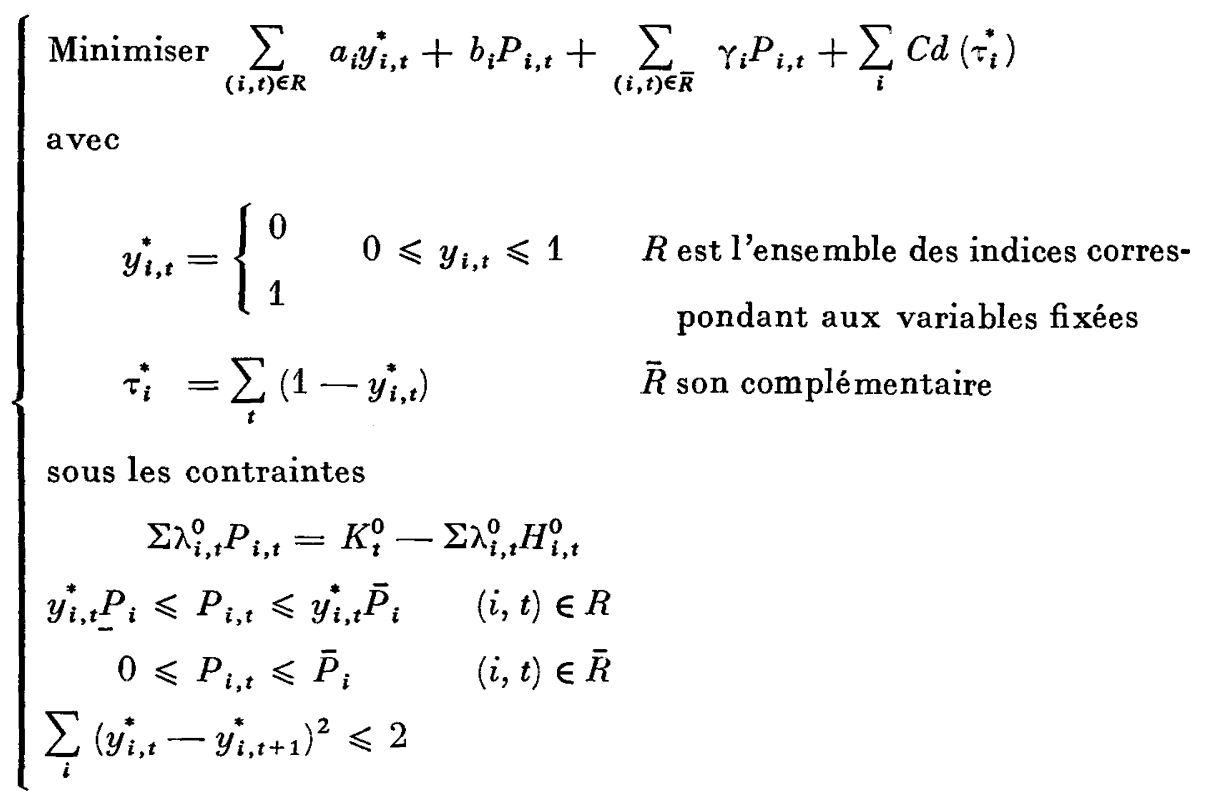

Les seules variables bivalentes qui apparaissent dans ce programme sont fixées. Les termes non linéaires de la fonction économique $C d_{i}\left(\tau_{i}^{*}\right)$ sont constants puisque $\tau_{i}^{*}$ est fixé. On obtient donc un programme linéaire dont la résolution est très simple. Sa structure est analogue à celui qui a été résolu en 3 . L'optimum fournit l'évaluation $V d$ cherchée.

L'évaluation par excès $V r$ est obtenue à l'aide d'une "bonne " solution réalisable de $(D)$ en ce sommet de l'arborescence. Poür trouver cette solution on choisit les groupes dont l'état de marche est déjà imposé en ce sommet; puis l'on complète à l'aide de nouveaux groupes dont l'état n'est pas encore fixé, dans l'ordre du rapport croissant de leur coût moyen au coût $\lambda_{i, t}^{0}$ de l'énergie au nœud $i$ à l'instant $t$. La contrainte d'un seul arrêt pour chaque groupe ainsi que les contraintes de réserve tournante dont on n'avait point tenu compte dans l'évaluation par défaut de l'optimum doivent être respectées.

L'état de tous les groupes étant alors connu, la puissance produite par chacun de ceux qui sont en marche est déterminée en les plaçant à leur minimum technique et en élevant leur puissance dans l'ordre du rapport croissant de leurs coûts marginaux $b_{i}$ au coût $\lambda_{t, i}^{0}$ de l'énergie jusqu'à satisfaire les contraintes :

$$
\sum_{i} \lambda_{i, t}^{0} P_{i, t}=K_{t}^{0}-\Sigma \lambda_{i, t}^{0} H_{i, t}^{0}
$$

sur chaque palier horaire.

Connaissant les deux scalaires $V d$ et $V r$ associés au sommet de l'ar- 
borescence, il est dès lors possible d'appliquer la procédure S.E.P. telle qu'elle a été décrite au paragraphe 3 .

Au critère principal d'arrêt qui est

$$
V d_{p} \geqslant V r^{*}
$$

où $V r$ est la meilleure solution trouvée à ce stade de l'exploration de l'arborescence, on peut ajouter les critères secondaires suivants :

a) Si la séparation en un sommet conduit à un nombre d'arrêt d'un groupe supérieur à 1 , il est inutile de poursuivre l'exploration dans cette direction de l'arborescence.

b) Si la séparation conduit à un arbitrage tel que la réserve de puissance des groupes ne satisfait pas la condition de réserve tournante sur chaque palier, il est également inutile de poursuivre l'exploration dans cette direction de l'arborescence.

De tels critères permettent d'accélérer notablement la procédure S.E.P.

\subsection{Optimisation de la répartition des puissances des groupes thermiques et hydrauliques, le planning thermique étant fixé}

Supposons qu'après avoir linéarisé les équations du réseau au voisinage d'une solution réalisable, on ait grâce à l'algorithme précédent, déterminé le planning qui optimise le programme linéarisé $(D)$.

Il s'agit dès lors d'optimiser un programme non linéaire continu qui a la forme suivante :

$$
\text { PM1 }\left\{\begin{array}{l}
\operatorname{minimiser} \sum_{t} \sum_{i} b_{i} P_{i, t} \\
P_{i, t}+H_{i, t}-C_{i, t}=\varphi_{i}\left(\theta^{t}\right) \quad \forall i, t \\
y_{i, t}^{*} P_{i} \leqslant P_{i, t} \leqslant y_{i, t}^{*} \bar{P}_{i} \quad \text { avec } y_{i, t} \text { fixé } \\
0 \leqslant H_{i, t} \leqslant \bar{H}_{i} \\
\sum_{t} H_{i, t}=E_{i} \quad \forall i
\end{array}\right.
$$

La méthode utilisée pour résoudre ce programme est une méthode de décomposition.

Tel que se présente le problème, seules les contraintes $\Sigma H_{i, t}=E_{i}$ lient les paliers horaires les uns aux autres. Supposons en effet que nous connaissions une solution réalisable $\left(H_{i, t}^{0}\right)$ qui satisfasse ces contraintes.

Optimiser le programme PM1 par rapport aux variables $\left(P_{i, t}\right)$ revient à optimiser indépendamment autant de programmes PM2 qu'il y a de paliers horaires. 


$$
\begin{gathered}
\text { P. MARtin et A. MERlin } \\
\text { Minimiser } F_{t}=\sum_{i} b_{i} P_{i, t} \\
\text { PM2 }\left\{\begin{array}{l}
P_{i, t}+H_{i, t}^{0}-C_{i, t}=\varphi_{i}\left(\theta^{t}\right) \quad \forall i \\
y_{i, t}^{*} P_{i} \leqslant P_{i, t} \leqslant y_{i, t}^{*} \bar{P}_{t} \\
\text { Les } y_{i, t}^{*} \text { sont fixés. }
\end{array}\right.
\end{gathered}
$$

Un tel programme n'est autre qu'un dispatching économique réalisé en (actif " seul. Sa résolution est connue.

Soit $\lambda_{i, t}^{0}$ la variable duale associée à une relation d'injection, variable duale définie à l'optimum du programme PM2.

L'interprétation économique de $\lambda_{i, t}^{0}$ qui résulte des conditions de Kuhn et Tucker écrites pour PM2 permet de poser

$$
\lambda_{i, t}^{0}=-\frac{\partial \hat{F}_{t}}{\partial H_{i, t(\boldsymbol{H}, t)}^{o}}
$$

où $\hat{F}_{t}$ représente l'optimum de $F_{t}$ pour $H_{i, t}^{0}$ fixé.

Dès lors la fonction

$$
\psi\left(H_{i, t}^{0}\right)=\sum_{t} \hat{F}_{t}\left(H_{i, t}^{0}\right)
$$

soumise aux contraintes :

$$
\begin{gathered}
\sum_{t} H_{i, t}^{0}=E_{i} \\
0 \leqslant H_{i, t}^{0} \leqslant \bar{H}_{i}
\end{gathered}
$$

est définie implicitement au point $\left(H_{i, t}^{0}\right)$ par sa valeur et la valeur de ses dérivées en ce point par rapport aux variables $H_{i, t}$

$$
\lambda_{i, t}^{0}=-\frac{\partial \hat{F}}{\partial H_{i, t}}
$$

D'où la résolution du programme linéaire PM3

$$
\begin{array}{r}
\text { Maximiser } \sum_{i} \sum_{i} \lambda_{i, t}^{0} H_{i, t} \\
\text { РM3 }\left\{\begin{aligned}
\Sigma H_{i, t} & =E_{i} \\
0 \leqslant H_{i, t} & \leqslant \bar{H}_{i}
\end{aligned}\right.
\end{array}
$$

L'algorithme de résolution de PM1 est dès lors le suivant :

1) Soit $\left(H_{i, t}^{0}\right)$ une solution hydraulique réalisable (c'est-à-dire satisfaisant aux contraintes de PM3). 
La résolution des programmes PM2 permet de trouver la meilleure répartition $\left(P_{i, t}^{(0)}\right)$ du thermique, la valeur $F^{(0)}$ de la fonction économique ainsi que les coûts $\lambda_{i, t}^{(0)}$ de l'énergie aux différents nœuds du réseau correspondant à cette solution $\left(H_{i, t}^{(0)}\right)$.

2) On optimise PM3; ce qui donne une nouvelle répartition $\left(H_{i, i}^{(n)}\right)$

3) On résout PM2; ce qui donne $F^{(n)},\left(P_{i, t}^{(n)}\right),\left(\lambda_{i, t}^{(n)}\right)$

Si $F^{(n)}<F^{(n-1)}$, on procède à une nouvelle itération en résolvant le programme PM3.

On pose $n=n+1$ et on va en 2 ).

Si $F^{(n)} \geqslant F^{(n-1)}$ on pose :

$$
H^{(n+1)}=\frac{1}{2}\left(H^{(n)}-H^{(n-1)}\right)+H^{(n-1)}
$$

$n=n+1$. Et l'on va en 3).

Lorsque toute variation du vecteur $H_{i, t}$ autour de $H_{i, t}^{(n)}$ conduit à un accroissement de la fonction économique $F$, on est à l'optimum de PM1.

\subsection{Influence des termes d'ordre supérieur à 1 dans les relations d'injec- tion sur le choix du planning thermique}

Dans toute la phase d'optimisation précédente, on a supposé :

a) Qu'une solution réalisable du problème ayant été trouvée, on linéarise les équations du réseau au voisinage de cette solution. L'optimisation d'un tel programme permet de trouver un planning thermique.

b) Ce planning étant fixé, on optimise la répartition des puissances thermiques et hydrauliques suivant l'algorithme précédent.

c) Il reste donc à prendre en compte l'influence de cette nouvelle répartition sur le planning obtenu, c'est-à-dire de tenir compte de la non-linéarité des équations du réseau dans la détermination de ce planning.

La méthode utilisée est analogue à celle qui est exposée en 4.3. Nous n'y reviendrons pas.

\section{UTILISATION DES RESULTATS}

La résolution de l'ensemble du programme mathématique fournit :

- pour les groupes hydrauliques, leur puissance à chaque heure ;

- pour les groupes thermiques, leur plan de démarrage et leur puissance ; 
- les variables duales associées aux relations d'injection en chaque nœud ; elles représentent le coût de l'énergie amenée à ce nœud, indication qui peut servir pour l'établissement d'une tarification horaire.

Un examen plus détaillé indique les influences respectives de la topologie du réseau ou des coûts de démarrage dans la répartition des transits en énergie et dans le choix des puissances horaires, problème très combinatoire.

Sur un réseau à 200 nœuds, le calcul total nécessite moins de 10 minutes sur CDC 6600.

\section{BIBLIOGRAPHIE}

[3]* E. M. L. Beaxe et R. E. Small, "Mixed Integer Programming by a Branch and Bound Technique $),$ Proceedings of the IFIP Congress 1965, vol. 2 (1965). A. H. Land et A. G. Dorg, "An Automatic Method of Solving Discrete Programming Problems 》, Econometrica, vol. 28, pp. 497-520 (1960).

MM. Benayoun, Donet, Nghiem, B. Roy, "Le programme Poligami », $S E M A$, décembre 1966.

[4] MM. Dauphin, Ferngold et Sponn, "Méthodes d'optimisation de la gestion des groupes d'un réseau électrique », E.D.F., Bulletin des Études et Recherches, Série B-no 1, 1966.

MM. Carpentier, Cassopoglem, Bexlou, Bouneau, Xirokostas, "Méthode des injections totales », IFORS, novembre 1968.

[5] MM. Siroux, Annet, Cosme et Dupoux, "Programme d'optimisation de lé production et du transport du Service des Mouvements d'Énergie d'Électricita de France 》, R.G.E., 76, p. 570, mars-avril 1967.

M. Scano, "Gestion à court terme d'un ensemble thermique hydraulique ", R.G.E., 76, mars-avri] 1967.

\footnotetext{
* Les chiffres entre crochets correspondent aux différents paragraphes de la note.
} 